\title{
Análisis de la distribución del servicio de educación primaria en el valle de Toluca, México
}

\author{
Sergio Franco MaAss* \\ Cecilia Cadena Inostroza**
}

\begin{abstract}
This paper describes the application of a Geographical Information System (GIS-SIG) to analyze the distribution of elementary schools in Toluca Valley. The application of the SIG allowed us to characterise the elementary education service as a function of three fundamental aspects: the distribution of the school-age population and the educational infrastructure; the analysis of the shortage and surplus of the service and the analysis of the spacial coverage. The results identify some of the problems of the distribution of the education service, e.g. gender inequity or inattention of rural zones, among others. This demonstrates the importance of the application of SIGs as helpful tools in decision taking.
\end{abstract}

Keywords: State of Mexico, Geographical Information Systems (GIS $S I G)$, elementary education, decision taking.

\section{Resumen}

El artículo describe la aplicación de un Sistema de Información Geográfica (SIG) para analizar la distribución de escuelas primarias en el valle de Toluca. La aplicación del SIG permitió caracterizar el servicio de educación primaria en función de tres aspectos fundamentales: la distribución de la población en edad escolar y de la infraestructura educativa; el análisis del déficit y superávit del servicio y el análisis de la cobertura espacial. Los resultados obtenidos permiten identificar algunas problemáticas en la distribución del servicio educativo: inequidad de género, desatención de las zonas rurales, entre otros. Con ello se demuestra la importancia de la aplicación de los SIG como herramientas de apoyo en la toma de decisiones.

Palabras clave: estado de México, Sistemas de Información Geográfica (SIG), educación básica, toma de decisiones.

* Centro de Investigación en Ciencias Agropecuarias de la UAEm, México. Correo-e: serfm@uaemex.mx

** El Colegio Mexiquense A.C., México. Correo-e: ccadena@cmq.edu.mx 


\section{Introducción}

Pese a su innegable relevancia como parte de los procesos de planificación territorial, la localización de equipamientos educativos es un aspecto poco estudiado en nuestro país. En el análisis de la distribución y en la nueva ubicación de sitios adecuados para la construcción de escuelas, suele suponerse que la decisión política de localización de un determinado equipamiento se encuentra sólidamente apoyada por una serie de estudios y consideraciones técnicas sobre el comportamiento y distribución de la demanda del servicio. Esto, sin embargo, difícilmente se cumple.

En el estado de México la construcción de escuelas primarias depende de la Secretaría de Educación, Cultura y Bienestar Social (SECyBS) y del organismo público descentralizado Servicios Educativos Integrados al Estado de México (SEIEM), ambos pertenecientes al Gobierno del Estado de México. Estos organismos vienen aplicando una serie de procedimientos para la ubicación de nuevos planteles educativos que, en términos generales, parten de las demandas expresas de grupos de vecinos u organizaciones sociales. En este contexto, persisten los problemas de limitada cobertura en las zonas rurales al tiempo que aparecen fenómenos de superávit en algunas zonas urbanas.

Esta problemática fue el punto de partida de una investigación en la que se abordó, como objetivo medular, desarrollar un procedimiento de análisis espacial que, mediante la aplicación de Sistemas de Información Geográfica (SIG), permitiera caracterizar la distribución del servicio de educación primaria en el valle de Toluca, coadyuvando con ello a mejorar la toma de decisiones en materia de localización de equipamientos educativos. En este documento se describen los principales aspectos metodológicos implícitos en el desarrollo de dicho sistema, así como los principales resultados de su aplicación. Se trata, en definitiva, de una propuesta técnica para coadyuvar en los procesos de análisis de la problemática educativa territorial y, por consiguiente, no aborda muchos otros aspectos medulares. Tal es el caso del análisis de los factores políticos, culturales, de factibilidad económica, etc. El SIG debe concebirse como una herramienta complementaria a muchos otros procesos de análisis. 


\section{Algunos antecedentes}

De acuerdo con el Artículo $3^{\circ}$ constitucional, la educación básica en México debe ser laica, gratuita y obligatoria. Ello implica contar con un servicio público de enseñanza unificado y coordinado que permita expedir y distribuir la función social educativa entre federación, estados y municipios (GF, 2001). En este contexto, se debe buscar ampliar la atención educativa hacia los grupos de población que han quedado excluidos de las actuales estrategias y cuya educación es condición necesaria para mejorar la calidad y equidad en los aprendizajes a lo largo de la vida (GEM, 2001a).

Como parte de las políticas de reformas estructurales y descentralización, el gobierno federal inició desde 1982 un proceso de descentralización de la educación hacia los estados. Con estas medidas se pretendía que el gobierno federal ya no fuese el responsable de esta función. Fue así como en 1992, a través del denominado Acuerdo Nacional para la Modernización de la Educación Básica (ANMEB), la federación transfirió a los estados del país la responsabilidad de los gastos de la educación primaria y secundaria (Rodríguez, 1999). Tal descentralización implicó la transferencia ${ }^{1}$ de instalaciones, personal y recursos para que a partir de entonces fuesen los gobiernos locales los que se encargasen de la responsabilidad de impartir educación y el gobierno federal sólo mantendría algunas funciones relativas a la coordinación de políticas educativas de orientación.

Una de las principales responsabilidades del sistema educativo en las entidades es acercar la educación a las localidades a través de la construcción de escuelas.

De acuerdo con el Gobierno del Estado de México, la demanda potencial del servicio educativo se concentra en los niveles de primaria y secundaria (y con la reforma a la educación preescolar, ahora también en ésta). La población que asiste a la escuela en dichos niveles, sin embargo, indica una limitada cobertura. Los mayores rezagos en materia educativa se observan en las zonas rurales y el equipamiento tiende a concentrarse en las localidades urbanas (GEM, 2001a). De acuerdo con datos de la SECyBs, en el año 2001 había en la entidad un rezago de 7,308 espacios educativos, correspondientes a 689 aulas, $80 \%$ de las cuales correspondían a la demanda de educación básica (Tavira, 2001).

${ }^{1}$ Sobre las dificultades que ha representado el proceso de descentralización educativa pueden consultarse: Cabrero y Mejía (1998), y Latapí y Ulloa (1997), entre otros. 
El proceso de descentralización en el estado de México tuvo, como característica principal, el que ya desde los años setenta se contara con un sistema escolar estatal, lo que permitió que en el momento de la transferencia de los servicios y procesos educativos, el gobierno tuviese menos dificultades para diseñar organismos que atendieran uno u otro sistema.

Con la intención de evitar los conflictos que provocaría la integración de los sistemas estatal y federal, en 1992, se creó el organismo público descentralizado Servicios Educativos Integrados al Estado de México (SEIEM) el cual tiene entre sus funciones "desarrollar, dirigir, vigilar y evaluar los servicios de educación básica y normal transferidos” (GE, 2001b). A partir de entonces tanto la secybs del Gobierno del Estado de México, como los SEIEM, son los responsables de los servicios educativos en la entidad.

Los SEIEM, como sistema paralelo, han intentado atender la nueva responsabilidad y adoptar (o asumir) las políticas del sistema estatal. Uno de sus principales objetivos ha sido ampliar la cobertura educativa, desarrollando una planeación que racionalice los recursos y dé prioridad a la atención del déficit (SEIEM, 2001). En este contexto se ha desarrollado el Sistema de Información para la Planeación (SIPP-2000), el cual persigue lograr mayor eficiencia y eficacia en la localización de los planteles educativos mediante la aplicación de tecnologías para la gestión de información geográfica.

El SEIEM ha aplicado una metodología para la ubicación de infraestructuras educativas que, en términos generales, comprenden tres grandes etapas (SEIEM, 2000): trabajo de gabinete; investigación de campo, y análisis y determinación de las propuestas. El contenido de estas tres etapas se menciona a continuación:

i. Trabajo de gabinete: acopio y análisis de información a fin de elaborar listados de localidades susceptibles a la localización de un centro educativo o en las cuales es posible tomar acciones para mejorar la prestación del servicio, tales como la creación de nuevas plazas de profesores. Esta etapa implica la elaboración de listados de centros educativos de nueva creación tomando en cuenta, fundamentalmente, las demandas expresas de grupos de vecinos u organizaciones sociales.

ii. Investigación de campo: visitas a las zonas deficitarias del servicio educativo, con la finalidad de obtener información 
socioeconómica y geográfica de las localidades propuestas, delimitar áreas de influencia, cuantificar el déficit por atender e identificar las parcelas adecuadas para la construcción de los equipamientos.

iii. Análisis y determinación de las propuestas: aplicación del SIPP para proponer alternativas de localización.

La SECyBS, por su parte, es el organismo gubernamental encargado de fijar y ejecutar la política educativa, cultural y de bienestar social del estado de México (GEM, 2001b). Para la expansión y creación de instalaciones educativas de nivel básico viene aplicando una serie de procedimientos de localización y asignación que incluyen:

i. Estudio de factibilidad: análisis de diversos aspectos geográficos y socioeconómicos de las localidades propuestas, delimitación de áreas de influencia y cuantificación actual y futura de la demanda educativa.

ii. Planeación y programación: concluido el estudio de factibilidad, se remite al Comité de Instalaciones Educativas del Estado de México (cieem), con el fin de planear y programar la construcción de la infraestructura (cieem, 2001).

iii. Aprobación: La Dirección de la Unidad de Planeación, Evaluación y Control Escolar procede a su revisión y aprobación.

Ante esto se plantean diversas interrogantes. La primera, y quizá más importante, es la carencia de un mecanismo para identificar el déficit real de equipamientos educativos. En efecto, los estudios de campo y factibilidad que se vienen realizando parten de una serie de demandas sociales que, sin cuestionar su legitimidad, no necesariamente reflejan la distribución real del déficit educativo. Los grupos sociales organizados inciden de manera significativa en los procesos de localización y construcción de los equipamientos, logrando que los organismos gubernamentales atiendan preferentemente sus demandas, minimizando las consideraciones técnicas y de cobertura en el servicio. ${ }^{2}$ Tal es el caso de algunos grupos dentro de una misma localidad y que por razones de religión o ideológicas, solicitan nuevas instalaciones. En estos casos

${ }^{2}$ La cobertura del servicio educativo se entiende como "el grado de incorporación de todos aquellos niños y jóvenes que por su edad o situación deberían estar inscritos en el sistema educativo formal” (Aguado y Arteaga, 1996: 67) 
se concede el permiso, previo estudio de factibilidad, pero sin considerar aspectos de déficit real (Tavira, 2001).

\section{Objetivos de la investigación}

El objetivo central de la investigación fue desarrollar una aplicación de sIG que permitiera el análisis más detallado de la distribución de las instalaciones educativas en el valle de Toluca, identificando la cobertura espacial de tales equipamientos. Mientras que la cobertura total se vincula con la eficiencia, la calidad y la igualdad de oportunidades (Aguado y Arteaga, 1996), la cobertura espacial tiene que ver con la eficiencia espacial de la localización (medida en función del volumen global de desplazamientos que el conjunto de la demanda debe efectuar para utilizar las instalaciones) y la justicia espacial (que indica la variabilidad de las distancias que separan a cada individuo de la instalación más próxima) (Bosque, 1997). Todo proceso de planeación educativa responsable requiere de considerar los aspectos relativos a la caracterización de la cobertura total, en este trabajo se aborda un objetivo que, aunque modesto, contribuye a dicha caracterización. les:

El diseño del sistema se centró en tres aspectos fundamenta-

i. Estimar la población no cubierta por el servicio educativo a partir del análisis de la relación entre la población en edad escolar, la población total por área geoestadística básica (AGEB) y el promedio de alumnos por aula y por profesor.

ii. Analizar, desde el punto de vista espacial, el comportamiento del déficit y superávit de instalaciones educativas.

iii. Analizar la cobertura espacial de las instalaciones educativas a partir de criterios normativos y de eficiencia espacial.

Una vez desarrollado el sistema, podría utilizarse por el sEIEM y la SECyBs como una herramienta de apoyo dentro de sus procesos de localización de establecimientos educativos. 


\section{Puesta en marcha del sIG}

\subsection{Obtención de la información temática}

Todo dato geográfico se caracteriza por su componente espacial y su componente temática. Desde el punto de vista temático, la investigación requirió contar con información sobre:

i. Los equipamientos educativos en la región;

ii. La población por AGEB (urbanas y rurales).

Para la caracterización de la infraestructura educativa existente en la región se consideró la información en el ámbito estatal contenida en el Catálogo de Escuelas proporcionado por el Centro de Investigación Educativa de la Escuela Normal (CIEEN) (que incluye la clave, nombre, municipio, localidad, número de alumnos, aulas y el número de escuelas a construir por municipio), así como la base de datos en los ámbitos estatal y federal que maneja la Unidad de Planeación, Evaluación y Control Escolar (que incluye clave de escuela, nombre, municipio, localidad, turno, tipo de escuela, hombres inscritos por grado, mujeres inscritas por grado, total de inscritos por grado, grupos por grado, número de salones en donde el director da clases, número de docentes, aulas existentes y en uso, y existencia de laboratorio y taller).

En lo que respecta a la información estadística de población por AGEB, se consultó el XII Censo General de Población y Vivienda (INEGI, 2000), del que se obtuvo, para cada AGEB la población total, la población de seis a 12 años, la población por sexo de seis a 12 años, y la población por sexo de seis a 12 años que sabe leer y escribir o que asiste a la escuela. ${ }^{3}$ Es importante destacar que la información de AGEB rurales se obtuvo de la agregación de los datos por localidad (INEGI, 2000). Una descripción más detallada sobre los procedimientos de creación de las bases de datos puede consultarse en Colindres y Urbina (2002).

\subsection{Obtención de la información espacial}

Un aspecto que determina en buena medida el tipo y alcances de cualquier análisis geográfico es la naturaleza espacial de las uni-

3 Dado que el Instituto Nacional de Estadística, Geografía e Informática (INEGI) publica la información agrupada en el rango de seis a 14 años, fue necesario solicitar los datos de seis a 12 años. 
dades de observación en que se expresa la información geográfica. Desde el punto de vista de la componente espacial, existen tres formas de concebir el territorio: como una distribución discreta y aleatoria de elementos puntuales; como un conjunto de nodos conectados por arcos o interacciones, conformando una red, o como la distribución continua del fenómeno a lo largo del territorio.

En lo que respecta a la expresión espacial de la información geográfica, la investigación requirió la elaboración de tres coberturas básicas:

i. Mapa de la distribución de establecimientos educativos;

ii. Mapa del sistema regional de comunicaciones;

iii. Mapa de AGEB urbanas y rurales.

\subsubsection{Obtención del mapa de distribución de establecimientos educativos}

La elaboración de un mapa con la localización precisa de los establecimientos educativos implicó enfrentar un importante problema. A pesar de que la información proporcionada por la Unidad de Planeación, Evaluación y Control Escolar de la secyBs incluía la dirección o localidad donde se ubica la mayoría de los establecimientos educativos, fue necesario obtener la ubicación geográfica precisa de cada uno de ellos. Ante esta situación se procedió a ubicar cartográficamente cada una de las escuelas para definir sus coordenadas de localización y proceder a la generación de un archivo digital de puntos. El trabajo de georreferenciación requirió, en algunos casos, la realización de recorridos de campo para verificar la ubicación de algunos establecimientos educativos. Resulta pertinente señalar que la aplicación de la tecnología GPS podría ser una alternativa interesante para que las instancias responsables de la educación en nuestro país tuvieran un mayor control sobre la ubicación y distribución de la infraestructura educativa.

La elaboración de un mapa de la zona de estudio con la representación de calles y carreteras se basó en la cartografía topográfica en formato digital del INEGI, escala 1:50,000. De los archivos correspondientes a las cuatro cartas que cubren la zona de estudio (E14-a37, E14-a38, E14-a47 y E14-a48) fue posible extraer la cobertura correspondiente a las vías de comunicación. Finalmente, mediante la aplicación de un software de SIG, fue posible 
realizar la limpieza digital, verificar la conectividad y generar la topología de líneas.

\subsubsection{Obtención del mapa de AGEB urbanas y rurales}

Para la generación del archivo de polígonos con la distribución de las AGEB fue necesario resolver un problema importante. El INEGI maneja de forma independiente la distribución de las AGEB urbanas y rurales y unir ambas bases resulta muy difícil, ya que los límites urbanos no coinciden con los límites rurales. Para resolver este problema, la única alternativa viable fue la edición manual, considerando que los límites urbanos tenían una mayor precisión. Una vez terminada dicha edición, fue posible aplicar un software de SIG para la limpieza digital y la generación de la topología de polígonos (véase el mapa 1).

\section{Mapa 1}

AGEB urbanas y rurales del valle de Toluca

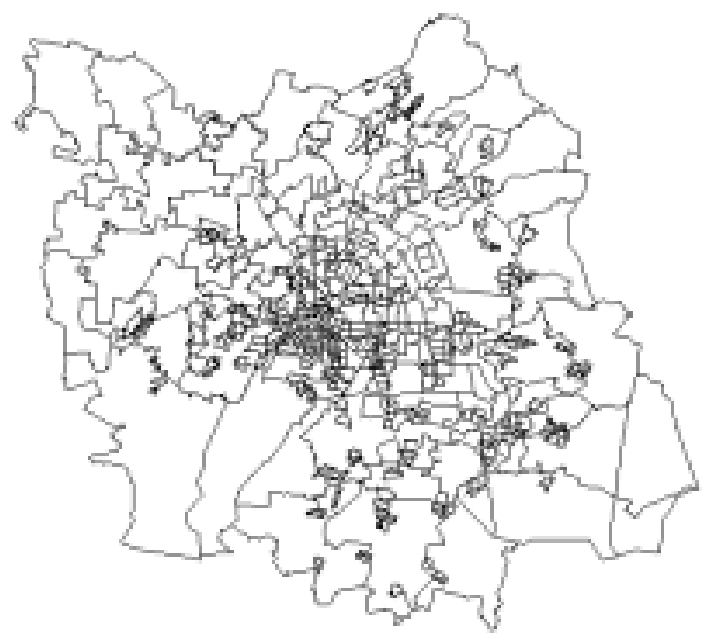

\subsection{Elección del tamaño del píxel}

Dado que algunos de los procedimientos de análisis requerían la utilización de un software de tipo raster, fue necesario, previo a la rasterización de los archivos, elegir el tamaño del píxel. Para establecer la resolución espacial, se consideró que la longitud del píxel o unidad base de la rejilla raster, debe ser la mitad de la longitud más pequeña que sea necesario representar de todas las existentes en la realidad (Bosque, 1997). 
De esta manera se definió un tamaño de píxel de 20×20 m. Esto, en definitiva, representaba una excelente resolución cartográfica, aunque afectaba seriamente el tamaño de los archivos, ya que implicaba generar coberturas raster de $22 \mathrm{Mb}$ (3,520 columnas y 3,179 renglones). Como se confirmó posteriormente, esto hizo más lenta la ejecución de algunos procesos, pero no obligó a redefinir el tamaño de píxel.

\section{Caracterización del servicio educativo en el valle de Toluca}

La aplicación de la tecnología SIG nos permitió caracterizar el servicio de educación primaria en el valle de Toluca en función de tres aspectos fundamentales: la distribución de la población en edad escolar y de la infraestructura educativa; el análisis del déficit y superávit del servicio y el análisis de la cobertura espacial.

\subsection{Distribución de la población en edad escolar}

En el año 2000 la población total de los municipios considerados ascendía a 1'741,984 habitantes, de los cuales 270,896 (15.56\%) correspondían a población entre los seis y los 12 años de edad, es decir, la población infantil en edad de cursar la educación primaria (INEGI, 2000).

\section{Mapa 2}

Población total de seis a 12 años por AGEB

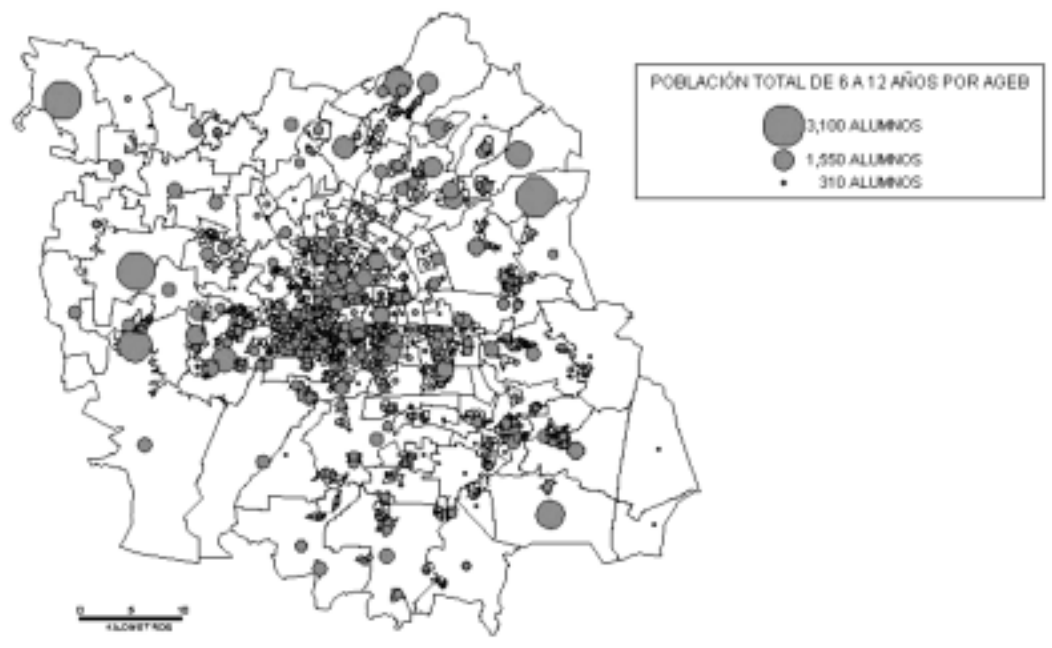


Como es posible observar en el mapa 2, la distribución de la población infantil es muy heterogénea en el territorio y tiende a aumentar en los AGEB rurales, especialmente en municipios como Almoloya de Juárez o Zinacantepec. Si bien es cierto que esto guarda cierta relación con el mayor tamaño de los AGEB rurales, también es un indicativo de la importante presencia infantil en las zonas periféricas.

Un mayor contraste se presenta en la distribución de la población infantil analfabeta (mapa 3). En este sentido, destacan los AGEB rurales sobre todo en municipios como Almoloya de Juárez, Tenango, Temoaya y Otzolotepec. Es importante destacar que para el año de referencia el analfabetismo regional alcanzaba $8.2 \%$ de la población en edades entre seis y 12 años.

\section{Mapa 3}

\section{Población de seis a 12 años analfabeta}

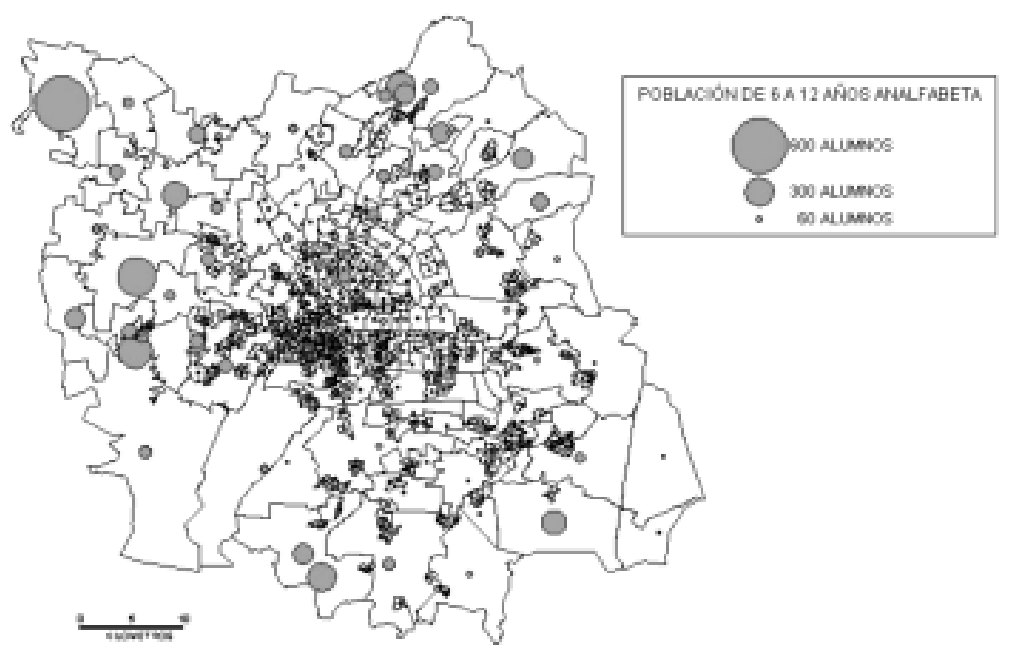

Ahora bien, en lo que respecta a la inasistencia escolar, ésta alcanzaba $4.3 \%$ de la población infantil de la región. En general, la asistencia escolar tiende a disminuir en regiones rurales apartadas, en especial en Almoloya de Juárez, Zinacantepec, Tenango y Otzolotepec (mapa 4). 


\section{Mapa 4}

Población de seis a 12 años que no asiste a la escuela

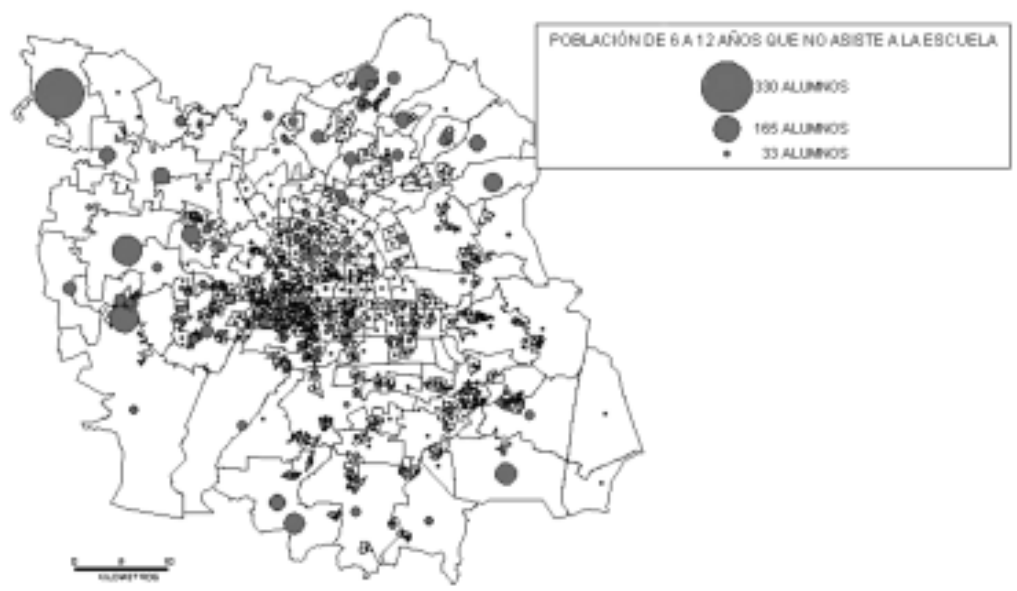

\subsection{Distribución de la población infantil por infraestructura educativa}

Un aspecto que resultaba de especial interés era el análisis de la asistencia escolar por sexo. En este sentido, se aplicó un índice específico [hombres/mujeres (que asisten a la escuela) x 100] para cada una de las zonas de influencia de los diversos centros educativos.

El mapa 5 permite observar la distribución de la asistencia escolar. Si bien es cierto que no es posible identificar un patrón de distribución tan claro en función de la proximidad a las zonas urbanas, es evidente la mayor participación del sector masculino en la mayor parte de los centros educativos. Destacan, en este sentido, el centro y suroeste de Zinacantepec, centro y este de Almoloya de Juárez y el oeste y noreste del municipio de Toluca.

En lo que respecta a la distribución de alumnos por aula, el promedio regional era de 26.7. Como se aprecia en el mapa 6, la mayor parte de los establecimientos educativos en las zonas rurales se encuentran por debajo de este valor y los promedios más elevados se presentaban en la ciudad de Toluca y sus alrededores. Esta realidad puede ser un argumento en contra del aumento de la construcción de instalaciones educativas en las zonas rurales periféricas. Es necesario, sin embargo, buscar esquemas alternativos que permitan la construcción de equipamientos más pequeños, pero con mayor cobertura. 


\section{Mapa 5 \\ Asistencia escolar por sexo}

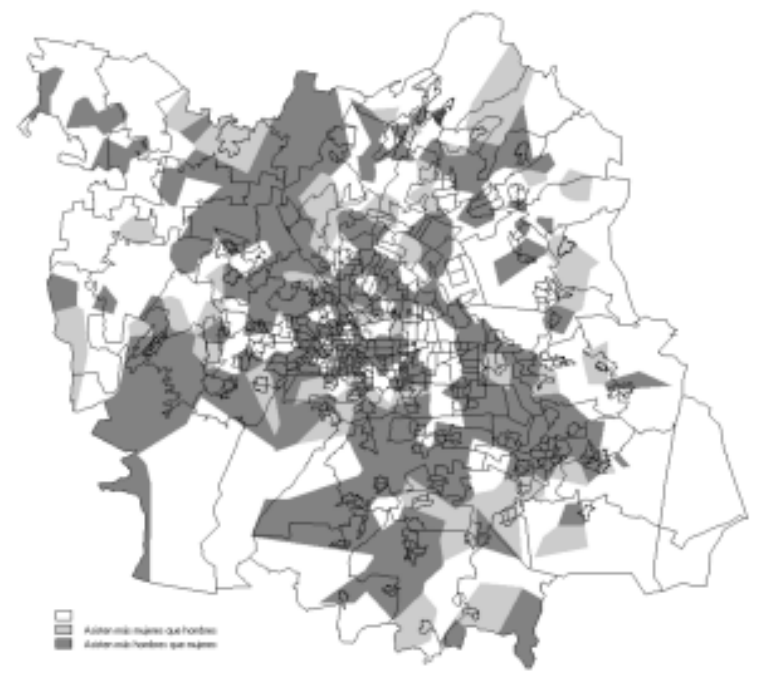

Mapa 6

Alumnos por aula (expresado en rangos)

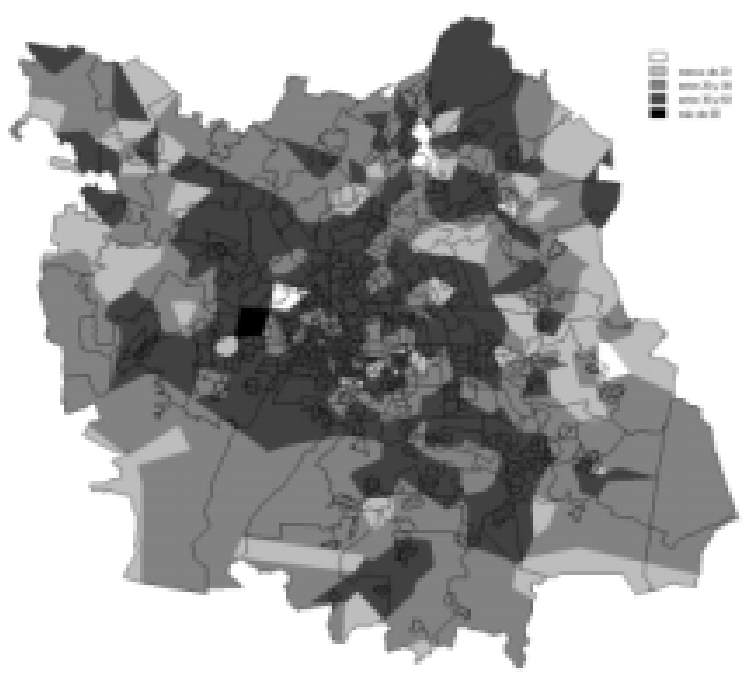

$\mathrm{Al}$ igual que en el mapa anterior, a mayor proximidad con la ciudad de Toluca, mayor es el promedio de alumnos por profesor. Los valores más bajos se presentan en municipios como Almoloya de Juárez, Otzolotepec, Tenango del Valle, Santiago Tianguis- 
tenco y Ocoyoacac. En el otro extremo, con valores muy elevados, destaca el centro de la ciudad de Toluca, donde se presentan casos de valores entre 51 a 60 alumnos por profesor (mapa 7).

\section{Mapa 7}

Promedio de alumnos por maestro (expresado en rangos)

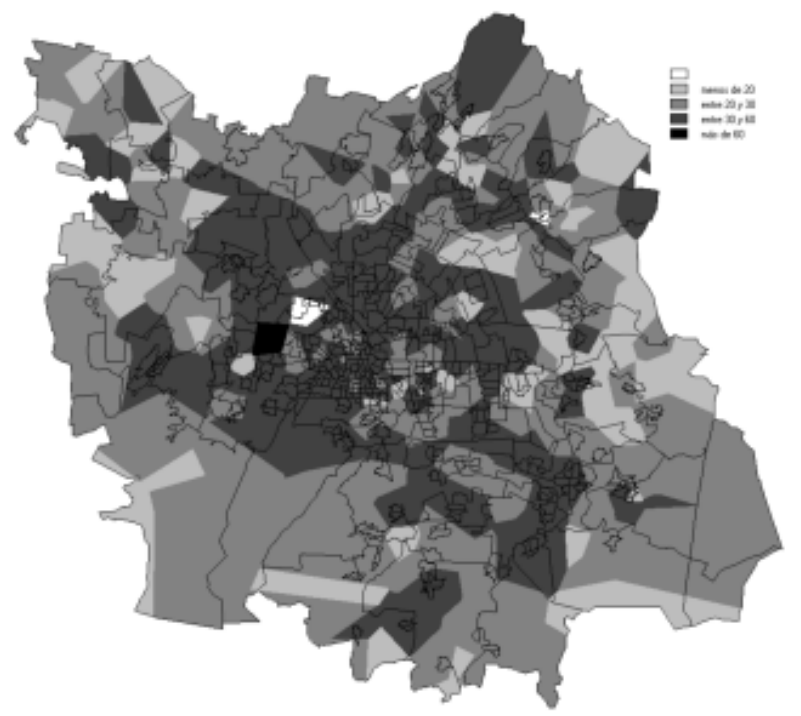

\section{Mapa 8}

Total de niños que debe atender cada centro educativo

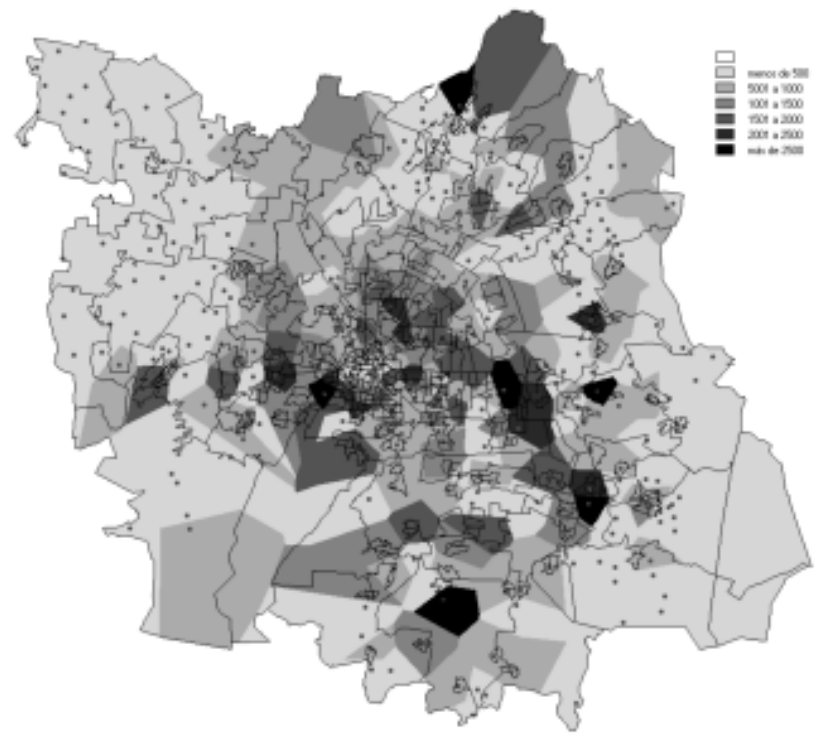




\subsection{Distribución de la demanda educativa}

Como resultado de relacionar la información por AGEB con el área de influencia de cada uno de los establecimientos educativos, fue posible obtener el déficit educativo para el año 2000. Como se observa en el mapa 8, el mayor déficit de educación primaria tendía a concentrarse en las cercanías a las zonas urbanas y en algunos AGEB dispersos por la región

\subsection{Distribución de la oferta educativa}

Para identificar el superávit educativo a nivel primaria, fue necesario extraer la información contenida en la base de datos por establecimiento educativo. Como se puede observar en el mapa 9 , la oferta educativa tiende a concentrarse en las zonas más pobladas. Destacan algunas regiones donde la oferta tiende a ser mayor: tal es el caso de municipios como Xonacatlán, Tenango, Joquicingo, Lerma, Ocoyoacac, Toluca y Zinacantepec.

\section{Mapa 9}

Total de niños que puede atender establecimiento educativo

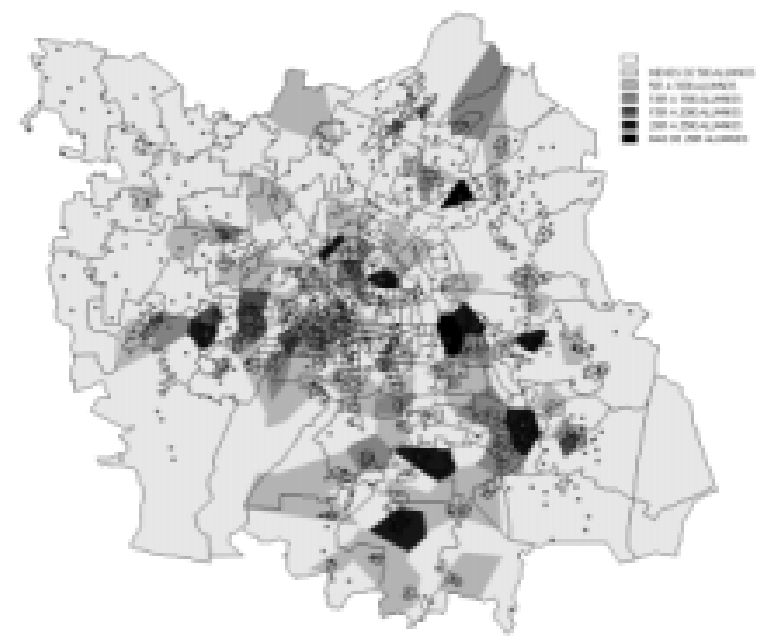

\subsection{Análisis entre oferta y demanda educativa}

Una vez caracterizadas la demanda y oferta educativas en la región, fue posible analizar comparativamente el comportamiento 
espacial del fenómeno. Esto implicó obtener un índice dentro del ambiente SIG.

Como es posible observar en el mapa 10, el déficit tiende a prevalecer con respecto al superávit (de acuerdo con los datos que arroja el sistema, cerca de 51 mil niños en edad de cursar la primaria podrían no estar siendo cubiertos por el servicio educativo). Un aspecto importante a destacar es el caso del municipio de Metepec, que aparece como altamente deficitario del servicio, pero, por los elevados niveles económicos imperantes en algunas zonas, la demanda se encuentra cubierta por establecimientos privados. Una tarea pendiente implica analizar la situación de los niños que viven en dicha región y que no pueden acceder a los sistemas privados de educación primaria.

Otro aspecto que es necesario señalar es el referente a los polígonos ubicados en los límites del área de estudio. En este caso, los resultados que se muestran en el mapa 10 no necesariamente reflejan la situación real. Cabe la posibilidad de que parte de la demanda educativa esté siendo cubierta por establecimientos educativos ubicados en regiones adyacentes.

En todo caso, los resultados nos permiten confirmar las disparidades regionales en cuanto a la distribución de la población infantil y de los equipamientos educativos. Destacan de manera significativa el caso de Almoloya de Juárez, que presenta condiciones muy dispares de déficit y superávit, y el caso del centro de la ciudad de Toluca, que presenta niveles muy elevados de superávit.

Mapa 10

Zonas con déficit o superávit de educación primaria (expresado en rangos)

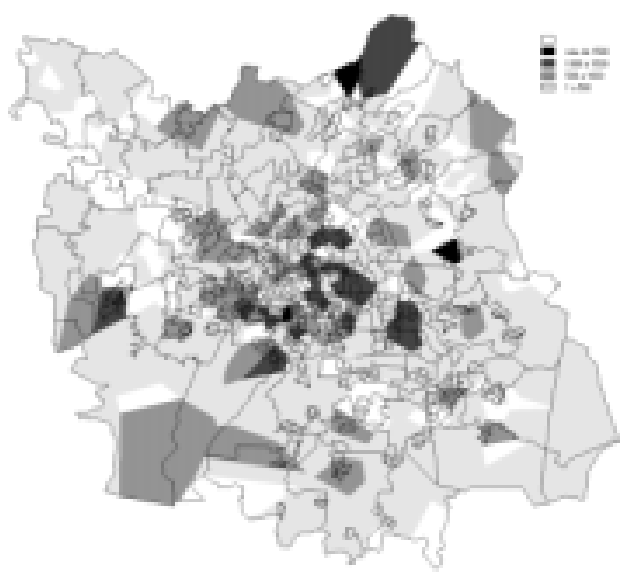




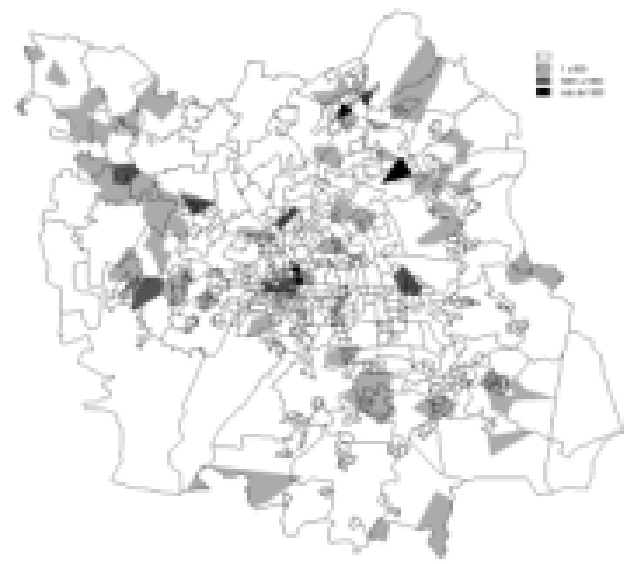

4.6 Diferencia entre aulas existentes y aulas en uso

Un aspecto que resultaba de interés en la investigación era determinar las posibilidades de cada establecimiento educativo para ampliar la cobertura del servicio sin la necesidad de construir nuevos equipamientos. Para ello, se consideró la existencia de diversas escuelas que reportan aulas en desuso. Como es posible observar en el mapa 11, los municipios de Tenango, San Antonio la Isla, parte de Zinacantepec, Toluca, San Mateo Atenco, Lerma y, en menor medida, la parte sur de Otzolotepec, tienen el mayor número de aulas no utilizadas.

\section{Mapa 11}

Aulas en desuso por equipamiento educativo

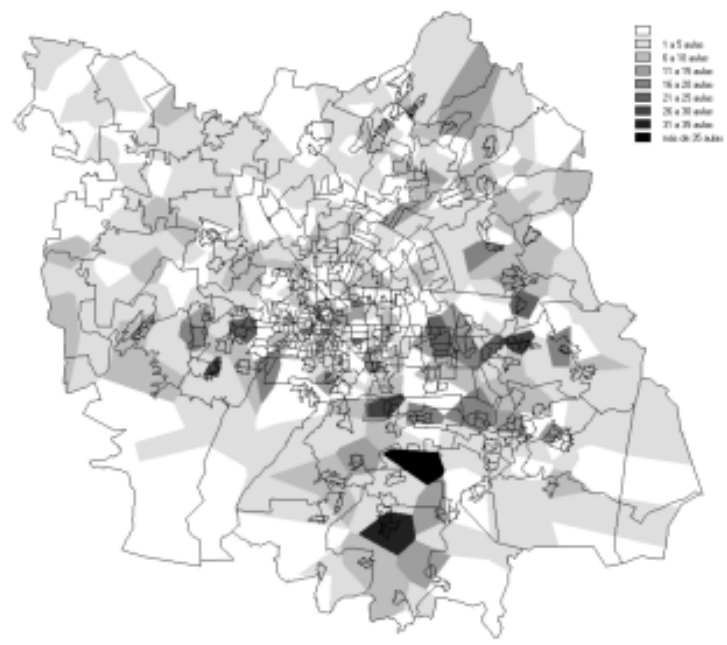


Como se ha mencionado previamente, de los cerca de 270 mil niños en edad de cursar la primaria, y dados los patrones de distribución de los establecimientos educativos, cerca de 51 mil podrían tener problemas de acceso. En este sentido, considerando el promedio regional de alumnos por aula (26.7), así como la distribución de los equipamientos con aulas en desuso, el sistema permitió calcular que, mediante la habilitación de dichos espacios, se podría atender a 21,866 niños más. Si bien es cierto que con esto no se cubriría el total de la demanda regional -ya que persisten los problemas de mala distribución de los equipamientos-, sí representaría una contribución significativa a la optimización de los recursos. El mapa 12 permite observar las regiones donde una política de habilitación de aulas en desuso podría tener un mayor impacto.

\section{Mapa 12 \\ Total de alumnos que se podrían atender con la habilitación de aulas en desuso}

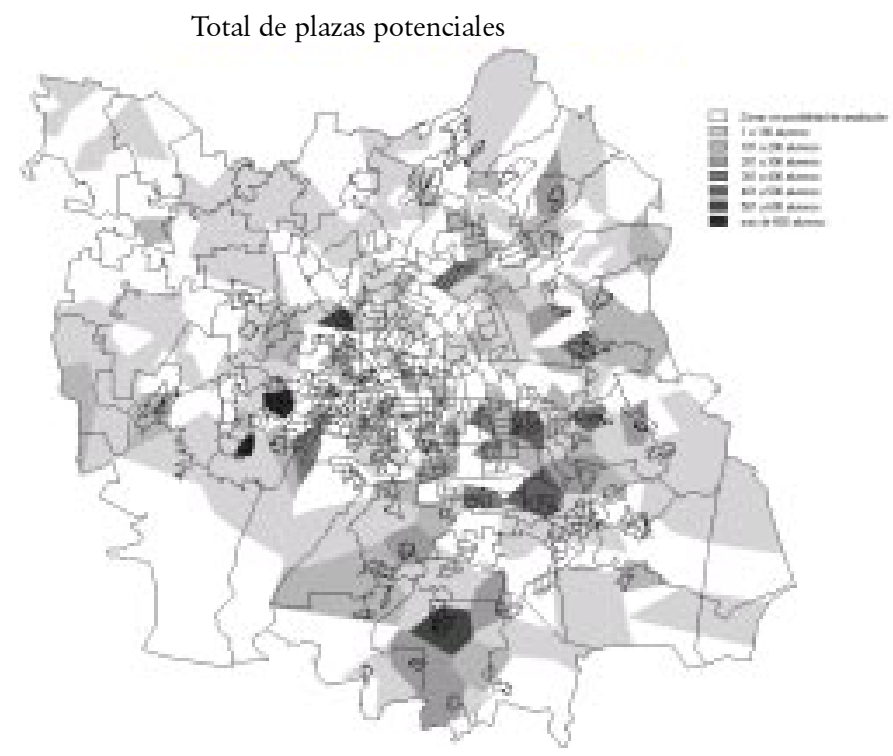

\subsection{Análisis de la cobertura espacial de los establecimientos} educativos

Como una forma de determinar las posibilidades reales que tiene la población para acceder al servicio educativo, se realizó un análisis de proximidad en distancia y tiempo de recorrido. 
4.7.1 Cobertura por proximidad en distancias lineales

Para efectos de estimar la población no cubierta por su lejanía con la escuela más cercana, se aplicó la normatividad de la Secretaría de Desarrollo Social que establece, como áreas óptimas de cobertura, $500 \mathrm{~m}$ en zonas urbanas y $5,000 \mathrm{~m}$ en zonas rurales (SEDESOL, 1995: 95). Tomando esto en consideración, fue posible derivar el mapa 13. Evidentemente, las zonas sin cobertura son básicamente rurales.

\section{Mapa 13 \\ Zonas de cobertura por proximidad en distancia a las escuelas primarias}

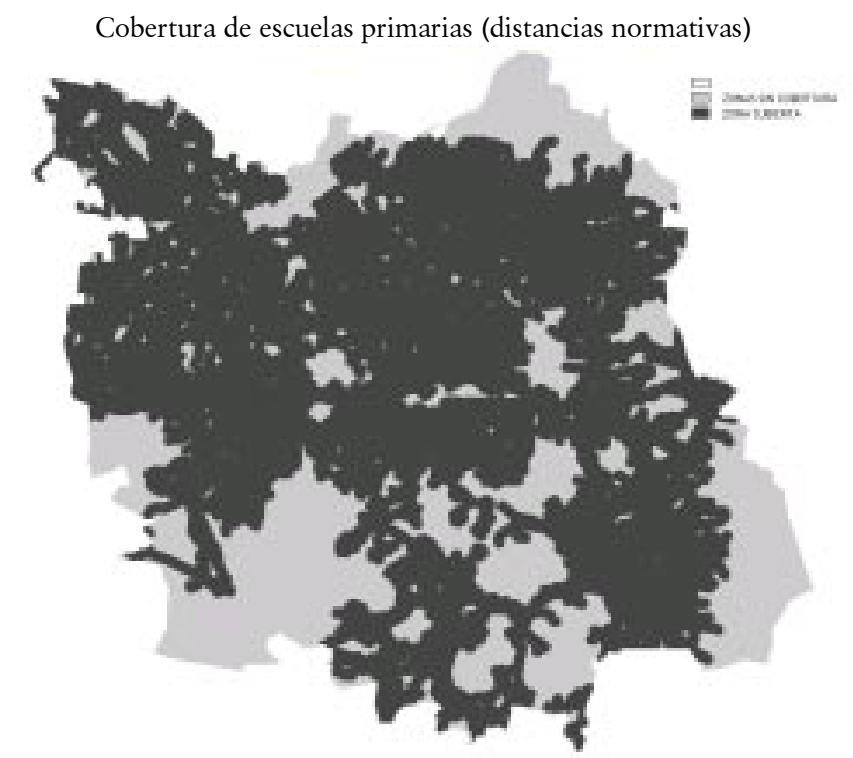

4.7.2 Cobertura por proximidad en tiempos de desplazamiento

Para realizar de este análisis se estimó el tiempo que un alumno debe tardar como máximo para llegar a la escuela más próxima. Para ello, se recurrió a la normatividad de SEDESOL (1995), que establece un tiempo máximo de 30 minutos en zonas urbanas y de 1 hora en zonas rurales.

Como es posible observar en el mapa 14, la distribución de las zonas sin cobertura guarda ciertas similitudes con el análisis basado en las distancias de recorrido. Sobresalen las zonas no cubiertas en la periferia de la región. Sin embargo, la diferencia fundamental radica en la dispersión de la cobertura en el municipio de 
Almoloya de Juárez, donde el sistema de comunicaciones es muy deficiente.

\section{Mapa 14 \\ Zonas de cobertura por proximidad en tiempo a las escuelas primarias}

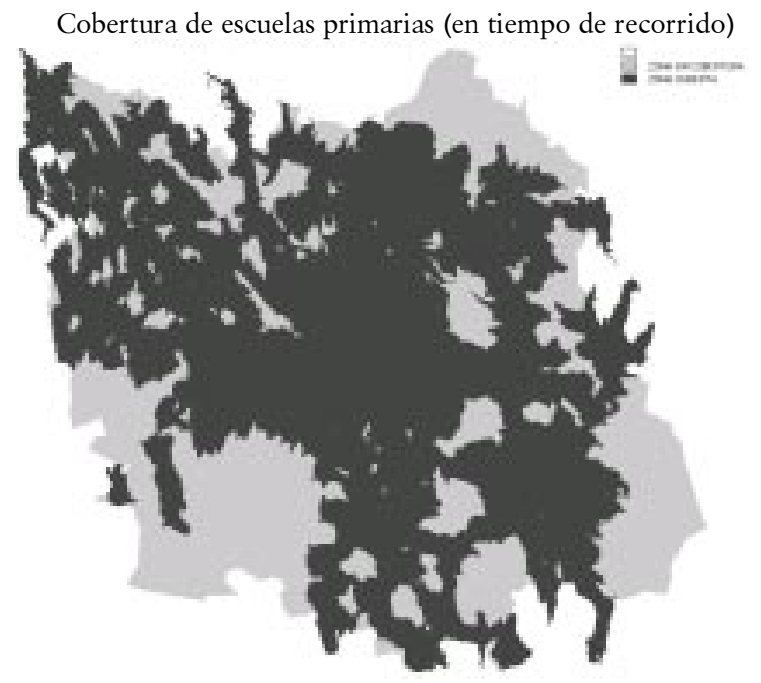

Tomando en cuenta el mapa de cobertura por proximidad en distancia (mapa 13), fue posible estimar que, para la fecha de referencia del estudio, podrían existir en la región cerca de 16,955 niños $(6.3 \%)$ habitando en zonas más allá de los límites normativos y que, por tanto, estarían enfrentando dificultades para acceder al servicio. Asimismo, al considerar el mapa de cobertura por proximidad en tiempo a los establecimientos educativos (mapa 14), se estimó que en la región existirían cerca de 15,889 niños $(5.9 \%)$ con dificultades para acceder al servicio.

\section{Conclusiones}

Mediante la aplicación de un SIG fue posible aplicar tres tipos de análisis: el cartográfico, el de déficit-superávit y el de cobertura espacial. De esta manera se logró una caracterización general de los servicios educativos de nivel primaria en el valle de Toluca.

La metodología utilizada no solamente permitió corroborar las enormes disparidades en la distribución de los servicios educativos regionales, sino que constituye un instrumento muy valioso para la planificación del sector educativo. Los SIG constitu- 
yen herramientas muy valiosas para el diagnóstico integral de los fenómenos educativos que, a partir del análisis en la distribución territorial de los fenómenos, nos permiten identificar los problemas del sector. Asimismo, mediante la aplicación de diversos modelos de simulación, es posible pronosticar el desenvolvimiento de los diversos fenómenos. Finalmente, la permanente actualización de estos sistemas facilita el monitoreo y seguimiento de las políticas educativas y las acciones gubernamentales.

Los resultados obtenidos muestran que, más allá de las dificultades financieras del proceso de descentralización aun no resueltas, persisten problemas de falta de una adecuada planeación y análisis en la toma de decisiones sobre la atención de necesidades educativas. Además de los esquemas tradicionales de localización de infraestructura, es posible identificar condiciones de subutilización, déficit y mala distribución espacial de los servicios educativos. La aplicación de los SIG para la evaluación de la cobertura educativa cuenta con antecedentes importantes. Los trabajos de Aguado (1995), Aguado y Arteaga (1996), Aguado (1997), Aguado (1998) y Aguado y Rogel (1999), son ejemplos muy valiosos. En ellos se aborda el análisis de diferentes variables relacionadas con la cobertura total o la así llamada "universalización de la educación básica" (Aguado, 1997). En todos estos casos se utiliza el AGEB como unidad territorial básica de análisis por considerar que "los indicadores agregados esconden profundas asimetrías entre espacios urbanos y rurales, y aun al interior de ellos" (Aguado, 1995: 185).

El presente trabajo retoma la necesidad de analizar información desagregada a nivel de AGEB. Estamos conscientes, sin embargo, que esto no resuelve totalmente el problema de la Unidad Espacial Modificable (UEM). En efecto, como lo señala Bosque (1997), el empleo de unidades de observación trazadas artificialmente tiene grandes repercusiones en los valores alcanzados por las variables, sin que realmente haya cambiado el valor subyacente del hecho temático. Se trata, en definitiva, de regiones artificiales y los resultados obtenidos no necesariamente representan al fenómeno estudiado.

A esto es preciso añadir que el problema detectado por Aguado (1995), en relación con la dificultad para integrar la información a nivel AGEB rural, no ha quedado totalmente resuelto. Coexisten en un mismo territorio grandes unidades de observación que agregan datos de localidades rurales y pequeñas unidades re- 
lativas a las AGEB urbanas. Evidentemente, las disparidades de superficie, implican serias distorsiones de la información.

Un aspecto a destacar es que, en el presente trabajo se intenta ir más allá en cuanto a la aplicación de los SIG. El planteamiento metodológico incluye el análisis de información expresada de manera coroplética (AGEB), pero se exploran algunos operadores de análisis de distancia proximidad a partir de la red de comunicaciones para evaluar la accesibilidad espacial en tiempo y distancia de recorrido.

La metodología utilizada, sin embargo, no ha estado exenta de problemas. El más importante de ellos fue la carencia de información adecuada que permitiera hacer un análisis más detallado:

i. Se carece de datos georreferenciados sobre la distribución de los establecimientos educativos. Esta es una tarea pendiente de los organismos oficiales;

ii. La información oficial no contiene el nivel de detalle requerido, situación que limita en buena medida los análisis;

iii. No existe coordinación entre las instituciones gubernamentales relacionadas con la enseñanza pública, lo que conduce a importantes lagunas de información, y sobre todo, al manejo de bases de datos incompatibles y con diferente nivel de detalle y actualización;

iv. La información estadística relativa a la población cuenta con importantes problemas de precisión y compatibilidad. Tal es el caso del Marco Geoestadístico, en el que no hay coincidencia entre límites de AGEB urbanos y rurales.

Si bien es cierto que existen procedimientos oficiales para la localización y asignación de nuevos centros educativos, éstos presentan serias desventajas, entre las que destacan las siguientes:

i. No se hace un análisis espacial de la distribución de los déficit y superávit del servicio educativo;

ii. No se consideran aspectos relacionados con la eficiencia y la equidad espacial, y

iii. No se toma en cuenta la normatividad que utiliza SEDESOL, específicamente en lo relacionado con los radios de cobertura de las escuelas existentes. 
Partiendo de lo realizado hasta ahora, resulta evidente la necesidad de desarrollar una metodología que, de forma complementaria, considere:

i. Realizar el análisis de cobertura total vinculada a las desigualdades socioeconómicas microrregionales;

ii. Aplicar otras técnicas de evaluación dentro del ambiente SIG, buscando mejorar la metodología desarrollada;

iii. Considerar otro tipo de variables como los factores geográficos de localización y que forman parte del proceso de acceso de los alumnos a los establecimientos educativos, y

iv. Utilizar diferentes modelos de localización y asignación, con el fin de minimizar distancias y mejorar su cobertura, obedeciendo a la justicia y eficiencia espacial.

\section{Bibliografía}

Aguado L., E. (1995), "La equidad, una asignatura pendiente: acceso y resultados educativos en cuatro zonas del Estado de México", en Pieck, G. E. y Aguado, L. E. (Coords.), Educación y Pobreza. De la desigualdad social a la equidad, El Colegio Mexiquense A. C. -UNICEF, Zinacantepec, México, pp. 183-235.

(1997), La universalización de la educación básica: ¿̇mito o realidad?, Documento de Investigación núm. 7, El Colegio Mexiquense A. C., Zinacantepec, México.

- (1998), Los problemas de la educación básica: el caso de San Felipe del Progreso. Documento de Investigación núm. 25, El Colegio Mexiquense A.C., Zinacantepec, México.

Aguado L., E. y N. Arteaga B. (1996), Diagnóstico de la Educación Básica en Naucalpan de Juárez. Hacia una propuesta de planeación microrregional, El Colegio Mexiquense A.C. y H. Ayuntamiento Constitucional de Naucalpan de Juárez 1994-1996, Zinacantepec, México.

Aguado L., E. y R. Rogel (1999), "La contribución de la educación a la justicia social: una ilusión fragmentada", en Civera, C. A. (coord.), Experiencias educativas en el Estado 
de México. Un recorrido histórico, El Colegio Mexiquense A. C., Zinacantepec, México, pp. 571-609.

Bosque, S. J. (1997), Sistemas de Información geográfica, Rialp, Madrid, pp. 227-230.

Cabrero y Mejía (1998), El estudio de las políticas descentralizadoras en México: un reto metodológico, CIDE-Miguel Ángel Porrúa, México.

Colindres, Isidro y Carlos Rogelio Urbina (2002), Propuesta metodológica para la aplicación de los SIG en el servicio público de enseñanza primaria y secundaria en la Región I (Toluca), Ciclo escolar 2000-2001, Tesis de Licenciatura en Planeación Territorial, UAEM, Toluca.

Comité de Instalaciones Educativas del Estado de México (CIEEM) (2001), Con Acciones Inmediatas el Estado de México Avanza 1999-2005, Gobierno del Estado de México, Toluca.

Gobierno del Estado de México (GEM) (2001a), Sistema de Información para la Planeación 2001-2002, documento en disco compacto, Gobierno del Estado de México, Toluca.

- (2001b), documento web http://www.edomexico.gob.mx/, consultado el 22 de mayo de 2001, México.

Gobierno Federal (GF) (2001), Constitución Politica de los Estados Unidos Mexicanos, (http://www.conggro.gob.mx/Federal/4.htm), consultado el 12 de junio de 2001, México.

Instituto Nacional de Estadística Geográfica e Informática (INEGI) (2000), Sistema de Información Censal 2000, INEGI, Aguascalientes, México.

Latapí, Pablo y Ulloa (1997), "El financiamiento de la educación básica en el marco del federalismo" (mimeo), CESU-UNAM, México. 
Rodríguez, Victoria (1999), La descentralización en México. De la reforma municipal a Solidaridad y el nuevo federalismo, Fondo de Cultura Económica, México.

Secretaría de Desarrollo Social (SEDESOL) (1995), Sistema Normativo de Equipamiento Urbano 1995, México, pp. 18-24.

Servicios Educativos Integrados al Estado de México (SEIEM) (2000), Sistema de Información para la Planeación (SIPP), documento en disco compacto, SEIEM, México.

- (2001), Servicios Educativos integrados al Estado de México, documento web http://www.seiem.gob.mx, consultado el 5 de junio de 2001, Toluca.

Tavira, Adriana (2001): Rezago Educativo, diario El Demócrata, 4 de julio, Pág. 11, Toluca.

Enviado: 29 de octubre de 2003.

Reenviado: 15 de marzo de 2004. Aprobado para su publicación: 27 de abril de 2004. 